\title{
Cannabis sativa var. indica : une plante complexe aux effets pervers
}

\section{Cannabis sativa var. indica : a complex plant with perverse effects}

Patrick MURA $^{(1) *}$, Bertrand BRUNET ${ }^{(1)}$, Yves PAPET $^{(1)}$, Thierry HAUET ${ }^{(2)}$

(1) Laboratoire de Biochimie et de Toxicologie, Centre Hospitalier Universitaire, BP 577 - 86021 POITIERS CEDEX - FRANCE

(2) INSERM ERM 324, Centre Hospitalier Universitaire - POITIERS - FRANCE

* Auteur à qui adresser la correspondance : Patrick MURA, Laboratoire de Biochimie et de Toxicologie, Centre Hospitalier Universitaire, BP 577 - 86021 POITIERS CEDEX - FRANCE Tél : 0549443923 - Fax : 0549443834 - E-mail : p.mura@chu-poitiers.fr

(Reçu le 15 janvier 2004 ; accepté le 9 février 2004)

\section{RÉSUMÉ}

Cannabis sativa var. indica appartient à la famille des Cannabinaceae et à l'ordre des Urticales. Parmi les 60 cannabinoïdes contenus dans la plante, essentiellement dans les feuilles et les sommités fleuries, le delta-9-tétrahydrocannabinol $(T H C)$ constitue le principal responsable des effets observables chez l'homme. La teneur en THC est très variable selon les conditions de culture, pouvant dépasser $20 \%$ dans le cas de cultures sous serre aux conditions parfaitement contrôlées. Après inhalation, le THC pénètre dans la circulation sanguine puis, très lipophile, va se fixer sur les tissus riches en lipides et en particulier au niveau du cerveau. Ses effets chez l'homme reposent sur l'existence des récepteurs $C B 1$ (essentiellement au niveau central) et $C B 2$ (surtout présents au niveau périphérique). Tandis que la présence du THC dans le sang n'est observable que pendant 2 à 3 heures après inhalation, des travaux très récents chez l'homme et chez l'animal nous ont permis de montrer qu'il restait fixé dans la plupart des structures cérébrales pendant de très nombreuses heures, cela expliquant la persistance importante de ses effets sur le système nerveux central. Les effets aigus sur le psychisme consistent principalement en des perturbations sensorielles, des troubles thymiques et dissociatifs, une diminution des performances intellectuelles, motrices et cognitives, des perturbations de la mémoire à court terme. Lors d'un usage important, régulier

\section{SUMMARY}

Cannabis sativa var. indica is a Cannabaceae, belonging to Urticales order. Among the 60 cannabinoids present in the plant and essentially in leaves and flowering tops, delta-9tetrahydrocannabinol (THC) is the main responsible of the effects observed in human using it. THC content is very variable depending on cultivation conditions, being able to contain more than $20 \%$ of THC in the case of under glass cultivations with well controlled conditions. After inhalation, THC moves to the blood and, because of its lipophily, distribution very fastly into the brain and other lipidic tissues. Its effects on human are supported by the presence of $C B I$ receptors (mainly in central nervous system) and CB2 (mainly present in other tissues). As THC is found in blood during only 2 to 3 hours after inhalation, recent studies allowed us to indicate that it remained present in brain structures during many hours, which explains the long persistence of its effects on central nervous system.

Acute side effects on behavior and central nervous system consist essentially in sensitive perturbations, thymic and dissociative troubles, a decrease of intellectual, motor and cognitive performances, short term memory disorders. For an important, regular and long term use, the occurrence of acute angor attacks or an amotivational syndrome are frequently observed. While risks for oneself related to cannabis use are not negligible, consequences for others may be exten- 
et prolongé, on note fréquemment l'apparition de crises d'angoisse aiguë et d'un syndrome amotivationnel.

Si les risques pour soi-même liés à son usage sont loin d'être négligeables, les conséquences pour autrui peuvent être considérables lorsque les consommateurs sont des conducteurs d'automobiles, des femmes enceintes ou des travailleurs occupant des postes à risque et/ou de sécurité en entreprise. L'importance du risque est majorée par un nombre de consommateurs de plus en plus grand. C'est pour cette raison que la France a récemment mis en place une législation destinée à sanctionner les conducteurs ayant fait usage de cannabis, sur la base des résultats de l'analyse sanguine. C'est aussi pourquoi il serait désormais opportun d'instaurer par voie législative des dépistages chez les personnes occupant des postes à risque et/ou de sécurité dans les entreprises.

\section{MOTS-CLÉS}

Cannabis, toxicologie, delta-9-tétrahydrocannabinol, THC.

\section{Introduction}

Les premiers témoignages de l'usage -de cannabis remontent à plus de cinq mille ans avant notre ère (1), ayant pris naissance sur les contreforts indiens et chinois de l'Himalaya (2). C'est avec les invasions arabes que la plante a commencé à faire son apparition en Europe. D'abord cultivée pour ses fibres qui servaient alors à la fabrication des voiles et cordages des navires, puis appréciée pour ses propriétés thérapeutiques (3), son utilisation à des fins récréatives ne prit son essor en France qu'à partir du milieu du XX ${ }^{\text {ème }}$ siècle. Si en 1940 on comptait à Paris moins de 10 usagers, c'est avec les mouvements hippies des années 60 que son usage commença à se généraliser. Aujourd'hui le cannabis est le produit psychoactif le plus consommé en Europe et dans la plupart des pays industrialisés. Les données françaises les plus récentes sont celles de l'enquête sur la santé et les consommations lors de l'appel de préparation à la défense (ESCAPAD) réalisée en 2001 par l'Observatoire Français des Drogues et Toxicomanies (OFDT) (4). Cette étude a révélé qu'à l'âge de 18 ans, $45,2 \%$ des filles et $55,7 \%$ des garçons déclarent avoir expérimenté le cannabis. $19,7 \%$ des garçons et $6,9 \%$ des filles avouent en consommer plus de 10 fois par mois. Par ailleurs, plusieurs enquêtes ont montré que cette consommation avait augmenté de manière très significative entre 1992 et 2002. Ainsi le rapport 2002 de l'OFDT indique que la prévalence de la consommation de cannabis au cours de la vie chez les 18-44 ans est passée de $20 \%$ à $35 \%$. Dans un tel contexte, il n'est pas étonnant que le cannabis soit devenu aujourd'hui l'objet de nombreux débats et controverses publics et politiques mais aussi scientifiques, avec des arguments qui ne sont pas toujours objectifs. C'est pourquoi les auteurs ont voulu par ce manuscrit apporter un éclaira- sive when consumers are car drivers, pregnant women or workers occupying a risk position. This risk is becoming very important today considering account of the number of consumers. Consequently, the French government and the Parliament have recently laid down a law, in order to punish drivers who would have consumed cannabis, based on the blood test results. It is also the reason for considering that it could be now advisable to set up legal controls for workers occupying a risk position.

\section{KEY-WORDS}

Cannabis, toxicology, delta-9-tetrahydrocannabinol, THC.

ge scientifique sur les dernières connaissances sur ce produit, en s'attachant à ne considérer que les éléments validés par la communauté scientifique internationale.

\section{Botanique}

Le cannabis n'est autre que le nom latin du chanvre (canapus), une plante appartenant à la famille des Cannabinaceae et à l'ordre des Urticales. Pendant très longtemps, on a distingué plusieurs espèces de chanvre : Cannabis sativa (chanvre textile), Cannabis indica (chanvre indien), Cannabis ruderalis, Cannabis sinensis. En fait la plupart des botanistes admettent aujourd'hui que toutes ces variétés ne sont que des chimiotypes différents de l'espèce sativa. Les différences observées dans la morphologie et la composition chimique sont consécutives à l'adaptation aux conditions climatiques. Cannabis sativa est le chanvre textile cultivé en Europe pour ses fibres (tissus, cordages) et pour ses graines oléagineuses ou chènevis. La plante peut atteindre deux ou trois mètres, et même jusqu'à six dans des conditions de culture idéale. Son aspect est tout à fait caractéristique (5). Il s'agit d'une plante herbacée, apétale, annuelle et le plus souvent dioïque mais parfois monoïque. Toutes les parties sont recouvertes de poils duveteux. La tige est droite, dressée, cannelée et plus ou moins ramifiée, s'implantant au sol par une forte racine pivotante et un système radicalaire développé (6). Les feuilles de la base sont opposées et 5-7 segmentées tandis que celles du sommet sont alternes, simples ou 3-segmentées ; les segments sont lancéolés et dentés (Figures 1 et 2). Les fleurs mâles sont réunies en panicules alors que les fleurs femelles sont groupées en cymes compactes, mêlées de bractées foliacées. Le fruit (le chènevis) est un akène ovoïde. 


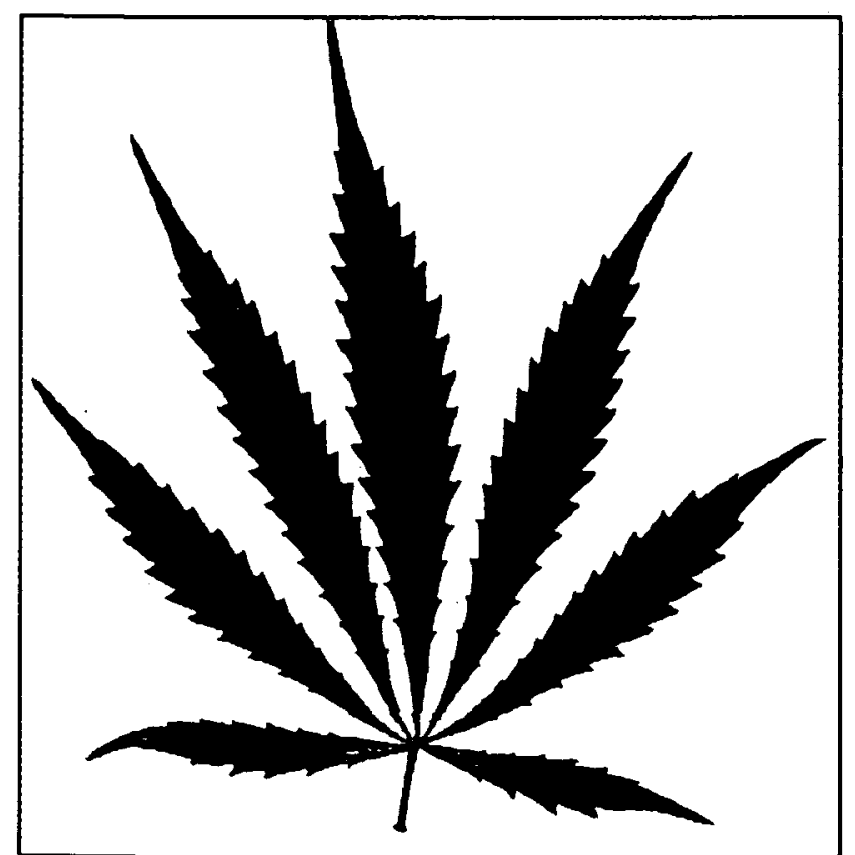

Figure 1 : Feuille de Cannabis.

\section{Principes actifs}

A côté des constituants classiques de très nombreuses plantes (flavonoïdes, terpènes, etc.), ont été recensés à ce jour plus de 60 cannabinoïdes. Parmi les principaux cannabinoïdes présents dans la plante et conduisant à des effets pharmacologiques chez l'homme, figurent : $>$ le $\Delta^{9}$-transtétrahydrocannabinol (THC), principal produit psychoactif chez l'homme (Figure 3).

$>$ le $\Delta^{8}$-transtétrahydrocannabinol, moins psychoactif que le précédent.

$>$ le cannabidiol.

$>$ le cannabinol, non psychoactif mais qui aurait une activité anti-inflammatoire.

$>$ les acides $\Delta^{8}$ et $\Delta^{9}$-tétrahydrocannabinoliques. Ce dernier n'est pas actif, mais est transformé en THC lors de sa combustion.

$>$ le cannabigérol (non psychoactif mais qui aurait une activité bactériostatique), le cannabichromène, le cannabicyclol, et leurs acides.

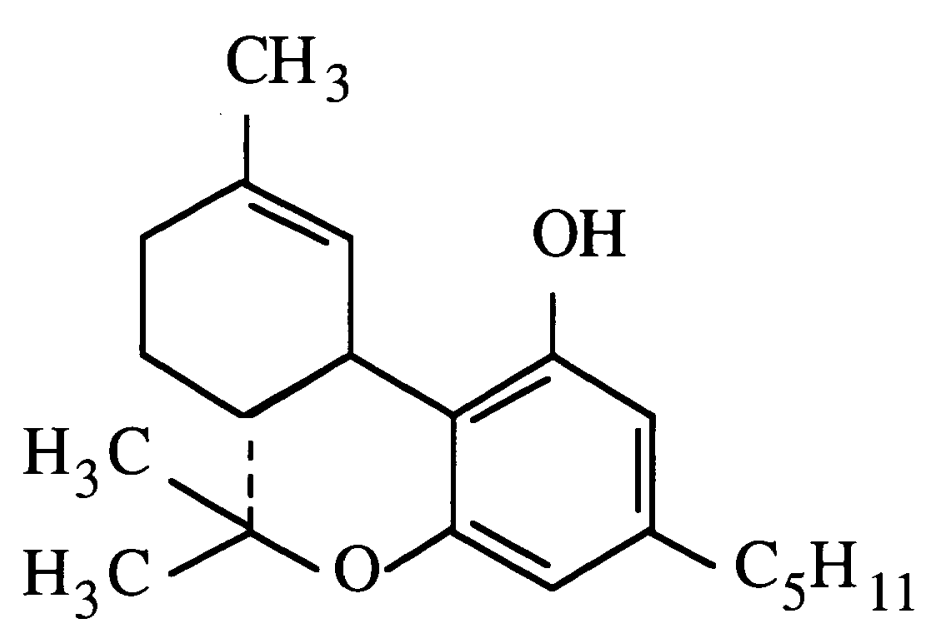

Figure 3 : Structure chimique du delta-9-tétrahydrocannabinol $(T H C)$.

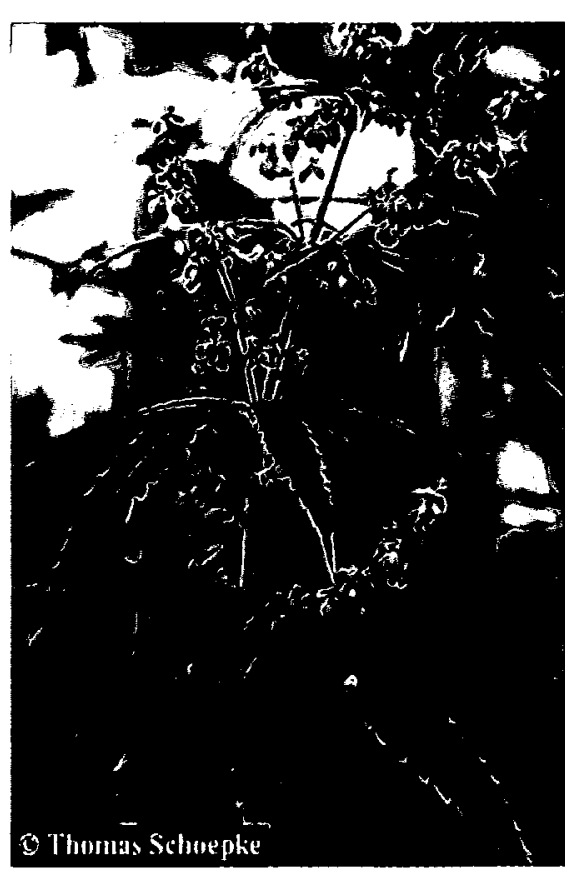

Figure 2 : Feuilles et somités fleuries de Cannabis (http://www.csdl.tamu.edu/FLORA/imaxxsol.htm)

Les teneurs en THC, en cannabidiol et en cannabigérol (pourcentage du poids sec) dans la plante peuvent servir à différencier les chimiotypes de Cannabis sativa (Tableau I).

D'une manière générale, la seule détermination du THC suffit pour différencier le type «drogue» du type «fibre». En Europe, la limite légale est de 0,2 \% de THC rapportée à la matière sèche (8).

Une étude récente a permis de montrer que les teneurs en THC dans les préparations à base de cannabis en France avaient augmenté ces dernières années, et principalement depuis 1996 avec notamment l'apparition de nouveaux produits très fortement titrés (9). Cette étude avait été réalisée sur 5152 résultats d'analyses obtenus de 1993 à 2000 sur des produits de saisies effectuées par les services des douanes, de la police nationale et de la gendarmerie nationale. Les résultats indiquaient que, quelle que soit l'année étudiée, une très grande variabilité des concentrations était observée pour «l'herbe» mais aussi pour la «résine». Des concentrations en THC inférieures à $2 \%$ étaient relativement fréquentes (18\% des échantillons). Jusqu'en 1995, $75 \%$ des échantillons d'herbe contenaient moins de 5,5\% de THC, et la teneur la plus élevée observée dans une saisie a été de $8,7 \%$. Pendant la même période, $47 \%$ des échantillons de résine contenaient entre 5

Tableau I : Détermination des différents types selon Fournier (7).

\begin{tabular}{|l|c|c|c|c|}
\hline Cannabinoïdes & \multicolumn{4}{|c|}{ Chimiotypes } \\
\hline & "drogue" & "intermédiaire" & \multicolumn{2}{|c|}{ "fibre" } \\
\hline delta-9-THC & $>2$ & $>0,5$ & $<0,3$ & $<0,1$ \\
\hline cannabidiol & 0 & $>0,5$ & $>0,5$ & $<0,1$ \\
\hline cannabigérol & & & $<0,1$ & $>0,5$ \\
\hline
\end{tabular}


et $10 \%$ de THC. Depuis 1996, les teneurs observées dans la majorité des échantillons n'ont pas beaucoup évolué : $54 \%$ des échantillons d'herbe et $71 \%$ des échantillons de résine contenaient respectivement moins de $5 \%$ et moins de $10 \%$ de THC. En revanche, sont apparus bien que de façon exceptionnelle des échantillons à base de cannabis très fortement concentrés en THC. En effet, au cours de l'année 2000, $3 \%$ des échantillons d'herbe et $18 \%$ des échantillons de résine analysés contenaient plus de $15 \%$ de THC. Les résultats concernant les échantillons de «résine» sont illustrés par la Figure 4.

\section{Présentations des produits et modes de consommation (10).}

L'herbe encore appelée «foin» ou «chiendent» est un mélange de sommités fleuries et de feuilles, séchées et réduites en poudre. C'est le «kif» du Maroc, la «marijuana» au Canada ou aux Etats Unis; le «dagga» d'Afrique du Sud, le «grifa» du Mexique ou le «takrouri» de Tunisie. La «sinsemilla» est une préparation de sommités femelles d'une variété privée de graines. Le «ganja» de l'Inde est composé uniquement de sommités fleuries fécondées et se présente soit sous forme aplatie par foulage aux pieds («flat ganja» ou «bombay ganja») soit roulée en magdaléons («round ganja» ou «bengal ganja»). Toutes ces préparations sont destinées à être fumées, mélangées à du tabac dans du papier à cigarette («joint») soit pures (pipes à kif). Certains consommateurs les fument en utilisant des narguilés, pipes orientales à long tuyau communiquant avec un flacon d'eau aromatisée que la fumée traverse avant d'être inhalée. Le «bangh» indien ou antillais est un mélange de tiges mâles et femelles et est utilisé sous la forme d'une boisson qui est le résultat d'un décocté de cannabis dans de l'eau ou de l'alcool.

Le haschich, encore appelé «hasch» ou «shit», est une poudre compacte brunâtre ou jaunâtre obtenue par battage et tamisage des feuilles et des sommités florales sèches, puis compressée sous forme de «barrettes». Il est le plus souvent mélangé à divers ingrédients comme le henné au Maroc et au Liban ou le curry au Pakistan. Il faut généralement 45 à $70 \mathrm{~kg}$ «d'herbe» pour faire un $\mathrm{kg}$ de haschich. Fumé en mélange avec du tabac, il est aussi consommé avec des aliments, incorporé dans des pâtisseries par exemple.

L'huile de cannabis est un liquide visqueux, brun-vert à noirâtre, d'odeur vireuse: Elle résulte de l'extraction

\section{Échantillons}

(\%)

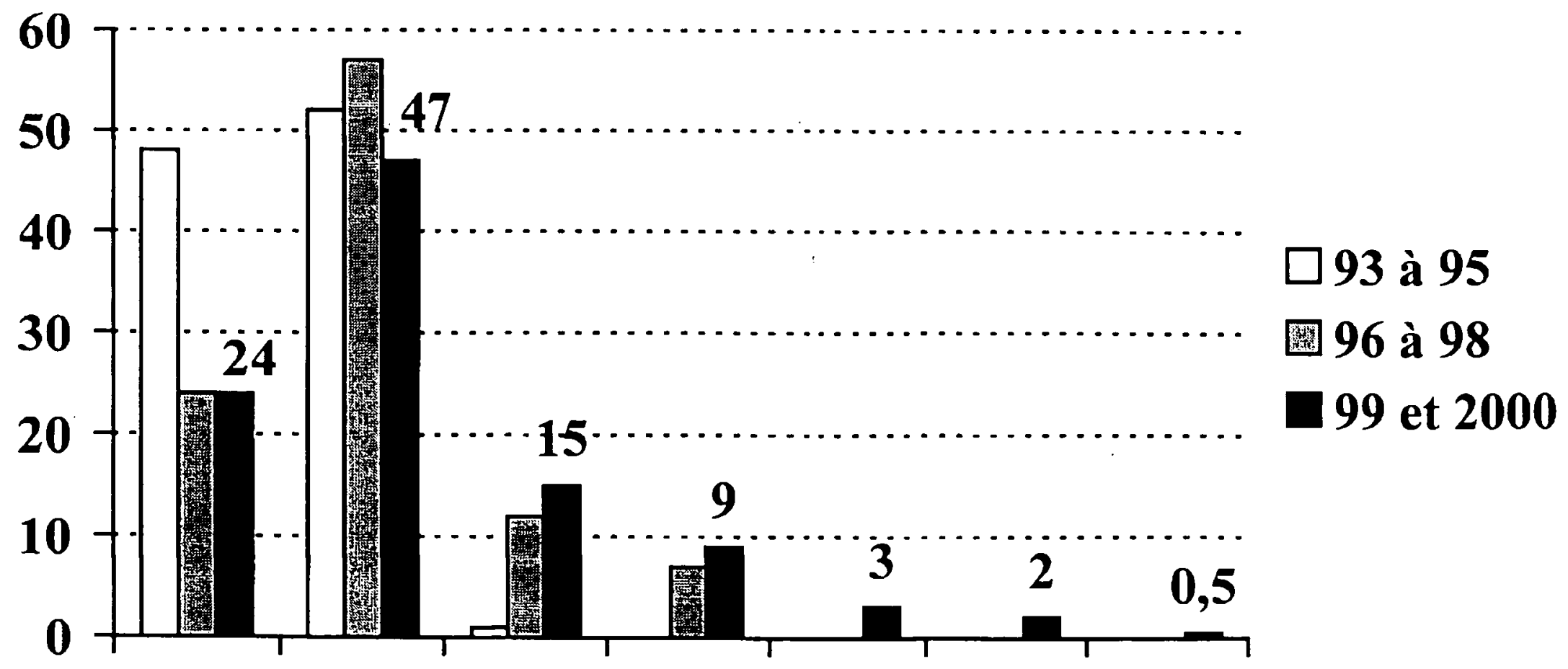

\section{$\begin{array}{llllllll}0-5 & 5-10 & 10-15 & 15-20 & 20-25 & 25-30 & >30\end{array}$ THC \% de matière sèche}

Figure 4 : Évolution des teneurs en THC observées en France pour la "résine" de 1993 à 2000 (9). 
de la résine par de l'alcool à $90^{\circ}$ suivie d'une exposition au soleil pour évaporer l'alcool. Le liquide ainsi obtenu est solidifié par chauffage afin de rendre le produit commercialisable. L'huile contient environ $60 \%$ de THC. Lorsqu'elle est consommée telle quelle, elle possède des effets hallucinogènes. Elle est destinée à être imprégnée sur du papier à rouler des cigarettes ou à être incorporée dans des préparations culinaires.

\section{Pharmacologie}

Après inhalation, 15 à $50 \%$ du THC présent dans la fumée sont absorbés et passent dans le flux sanguin (11). Les concentrations sanguines maximales (50 à $400 \mathrm{ng} / \mathrm{ml}$ ) sont obtenues en 7 à 10 minutes après le début de l'inhalation $(12,13)$ et sont dose-dépendantes. Très lipophile, le THC se distribue rapidement dans tous les tissus riches en lipides dont le cerveau, et de manière persistante dans toutes les zones cérébrales à forte densité de récepteurs (14). Le volume de distribution dans l'organisme est de 4 à $14 \mathrm{~L} / \mathrm{kg}$ (15). Cette fixation tissulaire importante est responsable d'une diminution rapide des concentrations sanguines (16). Des auteurs ont montré que $60 \mathrm{~min}$ après le début de l'inhalation d'un «joint» contenant $1,75 \%$ de THC, les concentrations sanguines étaient inférieures à $10 \mathrm{ng} / \mathrm{ml}$ (17).
Cette forte lipophilie, l'existence d'un cycle entérohépatique et de la réabsorption rénale se traduisent par des effets psychoactifs prolongés (18). Des auteurs (19) ont étudié sur simulateurs de conduite les effets (erreurs de conduite) obtenus par la consommation de «joints» dosés à 100,200 ou $250 \mu \mathrm{g} / \mathrm{kg}$ de poids du sujet. Ils ont indiqué l'existence d'une corrélation linéaire significative pendant une durée de 2 à 7 (selon les effets, le plus persistant étant le suivi de trajectoire). Les concentrations sanguines en THC ne peuvent être utilisées pour juger des altérations comportementales induites par une consommation de cannabis. En effet, sur la base des travaux de Cocchetto et coll (20), Harder et coll (21) ont comparé l'évolution dans le temps des concentrations sanguines de THC et les effets psychiques ressentis par les sujets, après consommation de «joints» contenant 1,3 ou $9 \mathrm{mg}$ de THC. Les résultats, illustrés par la Figure 5, montrent que les effets psychiques obtenus après consommation isolée d'un joint contenant $9 \mathrm{mg}$ de THC persistent pendant une durée d'environ $2 \mathrm{~h}$, tandis que la concentration en THC dans le sang est rapidement très faible et de l'ordre du $\mathrm{ng} / \mathrm{ml}$ au bout de $2 \mathrm{~h}$. Les mêmes auteurs ont par ailleurs montré que l'amplitude des effets était dépendante de la dose et de la concentration sanguine maximale observée.

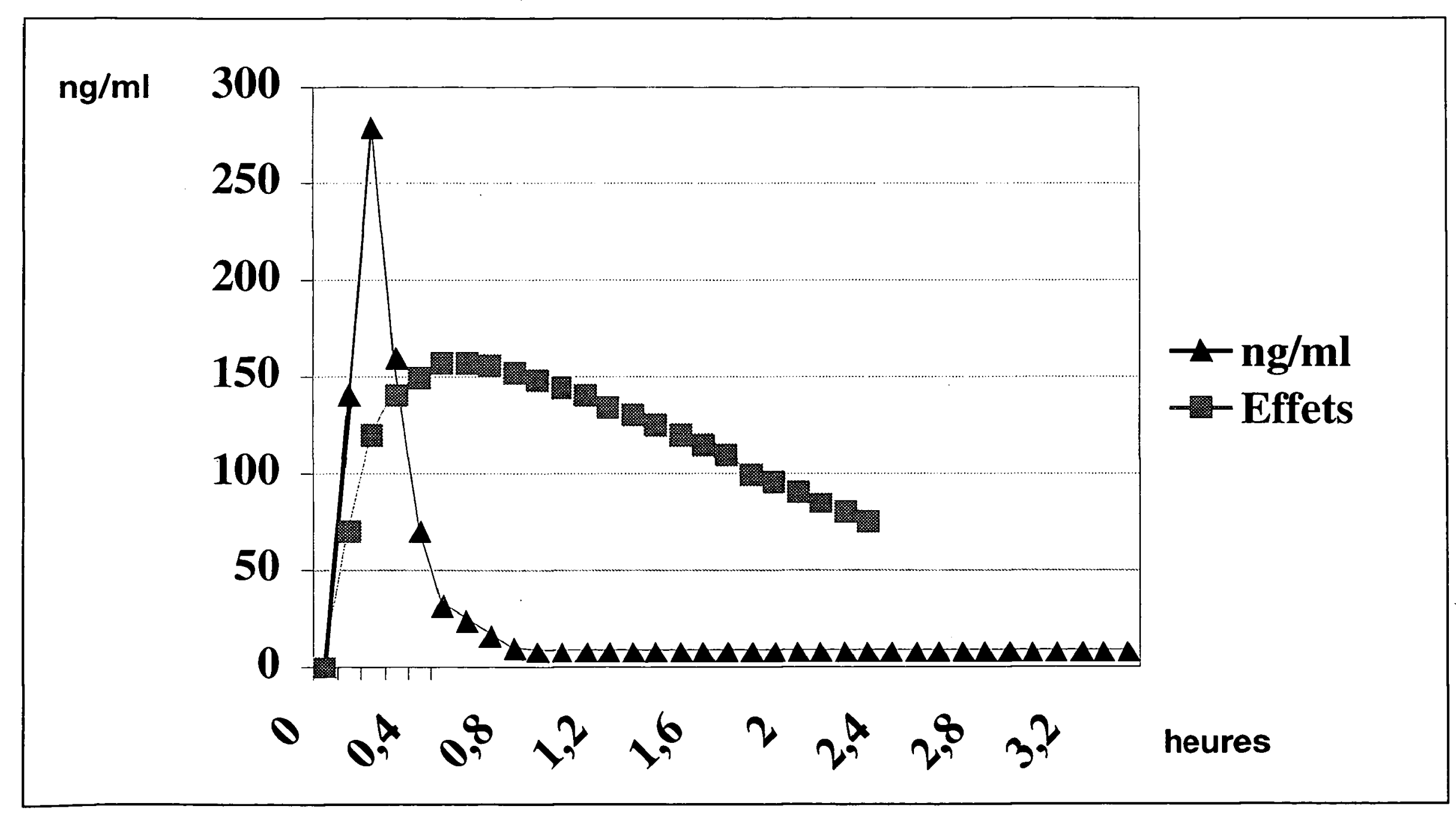

Figure 5 : Concent, ations en THC (triangles) et effets physiques et psychiques ressentis par le sujet (carrés) en fonction du temps après inhalation de $9 \mathrm{mg}$ de THC (20). 
Le THC subit, au niveau des microsomes hépatiques, un métabolisme oxydatif conduisant aux composés suivants :

$>$ le 11-hydroxy-tétrahydrocannabinol (11-OH THC). Métabolite psychoactif, ses concentrations sanguines sont de 4 à $20 \mathrm{ng} / \mathrm{ml}$ après 20 minutes et inférieures à $1 \mathrm{ng} / \mathrm{ml} 4$ heures après le début de l'inhalation (13). Des études chez l'animal (22) ont montré que son principal transporteur dans le sang était l'albumine tandis que le THC est essentiellement lié aux lipoprotéines. Lorsque le cannabis est consommé par ingestion, la quasi-totalité du THC est hydroxylée (principalement en 11-OH THC) au niveau de la muqueuse intestinale, ce qui se traduit dans le compartiment sanguin par une concentration en 11-OH THC supérieure à celle du THC. $>$ le $8 \beta$-hydroxy- $\Delta^{9}$-tétrahydrocannabinol, potentiellement psychoactif mais dont la participation aux effets du cannabis est négligeable en raison de ses très faibles concentrations et d'un métabolisme très rapide.

$>$ deux autres composés hydroxylés, dérivant des précédents et considérés comme inactifs, ont été identifiés : le 8-bêta,11-dihydroxy- $\Delta^{9}$-tétrahydrocannabinol et le 8-alpha-hydroxy- $\Delta^{9}$-tétrahydrocannabinol.

$>$ le 11-nor-9-carboxy- $\Delta^{9}$-tétrahydrocannabinol (métabolite acide, THC-COOH). Obtenu par oxydation du 11-OH THC, il ne possède aucune activité pharmacologique. Cet acide commence à apparaître dans le sang dans les minutes qui suivent l'inhalation. Au cours des étapes successives de distribution et de métabolisme du THC, les concentrations en THC-COOH dans le sang augmentent tandis que celles de THC décroissent (23).

Le schéma de ce métabolisme est illustré par la Figure 6 (24).

L'élimination des cannabinoïdes se fait par différentes voies : digestive, rénale, sudorale. Environ 15 à $30 \%$ du THC sanguin sont éliminés dans les urines sous forme de THC-COOH, et 30 à $65 \%$ le sont par les selles sous forme de 11-OH THC et de THC-COOH. En raison de sa forte fixation tissulaire, l'élimination urinaire est lente. Les demi-vies d'élimination sont très variables selon la dose et selon qu'il s'agit de consommateurs occasionnels ou réguliers. Par exemple, dans le cas d'une consommation peu importante mais répétée (un «joint» par jour contenant 1,75 ou $3,55 \%$ de THC pendant 2 semaines), elles sont comprises entre 44 et 60 heures (25). Chez de gros consommateurs réguliers, du THC-COOH peut être encore présent dans les urines 27 jours après arrêt de la consommation (26).

\section{Mécanismes d'action}

Les récepteurs et leurs localisations au niveau du cerveau sont désormais bien connus. Les récepteurs CB1 sont des récepteurs membranaires interagissant avec les protéines G, constitués de 473 acides aminés et comportant plusieurs sites de glycosylation. Ils sont localisés principalement au niveau central dans les structures suivantes : cortex frontal, cortex occipital, substance noire, cervelet, hippocampe. Des concentrations bien moindres de récepteurs $\mathrm{CB} 1$ ont aussi été retrouvées au niveau de l'utérus, des gonades, du cœur et de la rate. On n'en retrouve pas dans le bulbe, ce qui explique l'absence de décès directement imputable à une surdose de cannabis.

Le récepteur CB2 est aussi un récepteur membranaire. Absent du système nerveux central, on le retrouve surtout dans les éléments figurés du sang : lymphocytes $B$, lymphocytes T, monocytes «natural killers» (NK).

Les mécanismes impliqués dans la voie hédonique, concourant au sentiment de plaisir procuré par ces produits, sont aujourd'hui bien documentés, aboutissant notamment à une augmentation de la libération de dopamine au niveau du noyau accumbens et de l'aire tegmentale ventrale. De même, les mécanismes responsables des perturbations cognitives et motrices sont aujourd'hui bien décrits (27). La liaison des cannabinoïdes aux récepteurs $\mathrm{CB} 1$ entraîne une inhibition de l'adényl-cyclase par l'intermédiaire de la protéine Gi et une activation des AMP kinases par l'intermédiaire des sous unités $\beta 8$. Parallèlement, les cannabinoïdes modulent les canaux potassiques dans l'hippocampe et les canaux calciques de type $\mathrm{N}$ dans le ganglion cervical supérieur. Le ligand naturel de ces récepteurs est l'anandamide, un dérivé naturel de l'acide arachidonique. L'anandamide possède une bonne affinité pour les récepteurs $\mathrm{CB} 1$ et une affinité bien moindre pour les récepteurs CB2. Il diminue l'activité de la cellule nerveuse. Ce neurotransmetteur n'est pas stocké par les neurones mais réside dans leur membrane sous forme d'un précurseur phospholipidique, dont il est libéré sous l'influence d'une phosphodiestérase de type D.

\section{Effets aigus et chroniques chez l'homme}

En ce qui concerne les effets aigus, leur intensité varie selon la quantité consommée, la qualité du produit, l'ancienneté de la consommation (28), la tolérance du sujet, la structure de sa personnalité et son état d'esprit du moment, le contexte dans lequel s'inscrit la consommation. Bien entendu, ils varient aussi selon qu'il a été pris isolément ou associé à d'autres produits psychotropes.

Les principaux effets somatiques observables après une consommation isolée sont : 


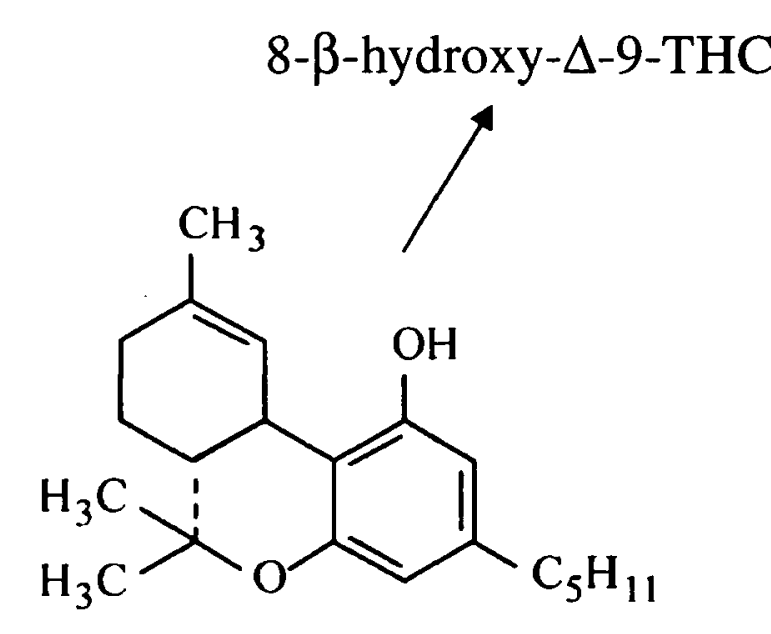

dérivés conjugués

$\Delta$-9-trans-tétrahydrocannabinol (THC)

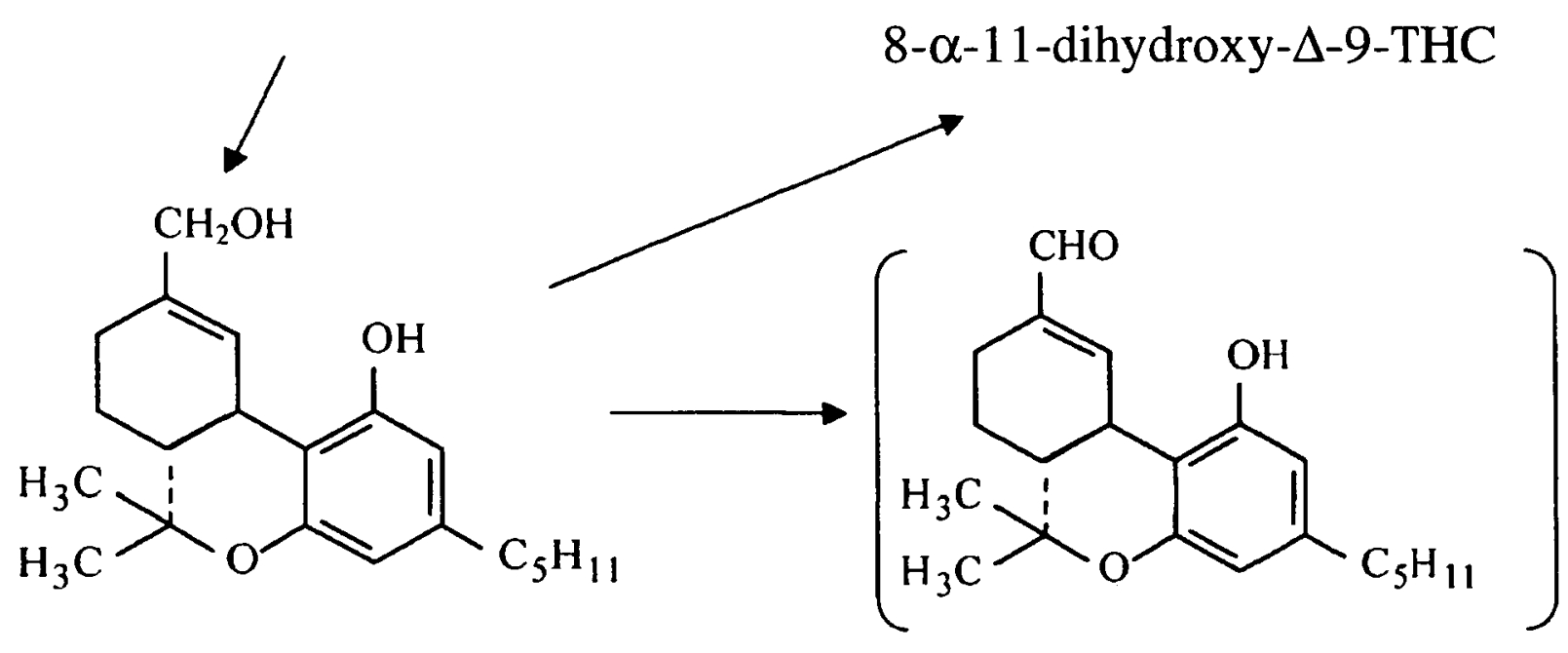

11-hydroxy- $\Delta-9-$ THC

11-oxo- $\Delta-9-\mathrm{THC}$

$\downarrow$

dérivés conjugués<smiles>[13CH3]</smiles><smiles>CCCCCCc1cc(O)c2c(c1)OC(C)(C)C1CCC(C(=O)O)=CC21</smiles>

11-nor-9-carboxy- $\Delta-9-$ THC

Figure 6 : Principales voies métaboliques du THC (24).

$>$ des effets cardio-vasculaires, consistant en une tachycardie et une hypotension orthostatique sans incidence sauf chez des sujets hypertendus ou porteurs d'une pathologie cardiaque.

$>$ une toxicité pulmonaire aiguë voisine de celle du tabac (29), avec la possibilité de réactions inflammatoires, de syndromes obstructifs, etc. Le THC induit par ailleurs une modification de la perméabilité alvéolaire se traduisant par une dilatation bronchique.
$>$ des signes digestifs fréquents. A forte exposition, et surtout lorsque le cannabis est ingéré, ils se traduisent par des vomissements, une diarrhée et des douleurs abdominales.

Les effets aigus sur le psychisme sont représentés par :

- des modifications de la perception du temps et des distances (30).

- des perturbations sensorielles (31) : perception exa- 
cerbée des sons et surtout des modifications de la vision associées à une mydriase, une diplopie et un nystagmus.

- une diminution de la vigilance associée à une sédation pouvant aller jusqu'à la somnolence, voire l'endormissement.

- des troubles thymiques et dissociatifs avec euphorie, anxiété, agressivité, dépersonnalisation avec disparition des inhibitions et indifférence vis-à-vis de l'environnement, une conscience accrue de soi.

- des hallucinations et délires possibles notamment avec les nouveaux produits pouvant être très concentrés en cannabinoïdes (9).

- une diminution des performances intellectuelles, motrices et cognitives.

- des perturbations de la mémoire immédiate pouvant persister après plusieurs jours, voire semaines d'abstinence.

- des crises d'angoisse aiguë, bien qu'exceptionnelles, au cours desquelles un véritable état de panique peut s'installer.

Parmi les effets somatiques imputables à une consommation régulière et importante, on note principalement :

- Des effets sur les fonctions de reproduction. Si les effets néfastes du cannabis sur la fertilité masculine et en particulier sur la production de testostérone sont actuellement sujets à controverses (32), il est admis qu'une consommation régulière par les femmes enceintes se traduit généralement par une diminution de la taille et du poids des fœtus (28).

- Des effets sur le système immunitaire. Le THC agit sur les cellules lymphoïdes comme un immunomodulateur. Il augmente la production ou la sécrétion d'interleukine-1 tandis qu'il inhibe fortement la production d'interleukine-2 et d'interféron, supprimant l'activité des cellules Natural Killer et inhibant la blastogenèse induite par les antigènes. Il a été démontré chez l'animal que ces propriétés conduisent à la suppression de la production d'anticorps et de certaines cytokines tandis que d'autres cytokines comme les cytokines proinflammatoires sont produites en excès. Toujours chez l'animal, l'administration de THC favoriserait de façon très significative la survenue d'infections bactériennes et virales (33).

- Un pouvoir cancérogène de la fumée de cannabis supérieur à celui de la fumée de tabac. Ce risque de cancérisation de la muqueuse bucco-pharyngée ou des tissus bronchique et pulmonaire est lié à une concentration importante dans la fumée de cannabis de substances particulièrement carcinogènes tels que le benzopyrène ou le benzanthracène (34). Ainsi par exemple, la teneur en benzopyrène est $70 \%$ plus élevée dans la fumée de cannabis que dans la fumée de tabac. Ce pouvoir cancérogène est augmenté par l'effet bronchodilatateur du THC, qui induit une rétention accrue des goudrons dans les alvéoles pulmonaires.

Les effets chroniques sur le psychisme sont dominés par :

- Un syndrome amotivationnel, fréquemment observé.

- Des syndromes de désorganisation de la pensée, psychotiformes, souvant survenir même chez des sujets sans antécédent psychiatrique.

- Une augmentation du risque de développement ou d'aggravation de la schizophrénie (35), ainsi qu'un risque suicidaire augmenté chez les schizophrènes consommateurs de cannabis (36).

\section{Identification et dosage}

La recherche et le dosage des cannabinoïdes occupent une part de plus en plus importante dans l'activité de certains laboratoires. Parmi les indications actuelles ou potentielles, on note les accidents de la circulation routière, les circonstances à caractère médico-légal, le dépistage des toxicomanies en milieu professionnel, le dopage, le suivi des patients toxicomanes sous traitement de substitution, etc...

Les milieux biologiques utilisés. Les cannabinoïdes, comme les autres stupéfiants et la plupart des xénobiotiques, sont retrouvés dans de nombreux milieux biologiques : sang, urine, salive (essentiellement par séquestration bucco-dentaire induite par l'inhalation), sueur, bile, cheveux et autres phanères. La nature du prélèvement à réaliser diffère selon le contexte en fonction du délai recherché et selon la nécessité ou non d'apprécier quantitativement le degré d'imprégnation (tableau II).

Tableau II : Principales caractéristiques des différents milieux biologiques utilisables pour la recherche ou le dosage des cannabinoïdes (d'après (27)).

\begin{tabular}{|c|c|c|c|}
\hline URINES & $\begin{array}{c}\text { Cannabinoìde(s) } \\
\text { majoritaires }\end{array}$ & $\begin{array}{c}\text { Délai } \\
\text { maximum de } \\
\text { détection }\end{array}$ & Domaine d'intérêt \\
\hline SALIVE & THC-COOH & $\begin{array}{c}\text { Consommation } \\
\text { occasionnelle : } \\
2 \text { à } 7 \text { jours } \\
\text { régulière : } \\
7 \text { à } 21 \text { jours }\end{array}$ & $\begin{array}{c}\text { Dépistage d'une } \\
\text { consommation }\end{array}$ \\
\hline SUEUR & THC & THC à 10 heures & $\begin{array}{c}\text { Dépistage d'une } \\
\text { consommation récente }\end{array}$ \\
\hline CHEVEUX & THC & $\begin{array}{c}\text { Environ } 1 \text { mois } \\
\text { pour chaque cm } \\
\text { de cheveux }\end{array}$ & $\begin{array}{c}\text { Révélation et suivi } \\
\text { dans le temps d'un } \\
\text { usage régulier }\end{array}$ \\
\hline SANG & THC-COOH & THC & Peu d'intérêt \\
(11-OC THC & à 10 heures & $\begin{array}{c}\text { Confirmation } \\
\text { Identification, } \\
\text { Dosage }\end{array}$ \\
\hline
\end{tabular}


Le dépistage immunochimique utilise principalement les techniques de type EMIT et FPIA, deux méthodes se différenciant par leur mode détection mais dont les performances sont très voisines. Selon les réactifs, les limites de détection sont de 20, 25, 50 ou $100 \mathrm{ng}$ de THC-COOH par ml d'urine. Ces réactifs sont utilisables sur des appareils automatisés ou semi-automatisés. Le risque de résultat positif après inhalation passive est fréquemment évoqué. En réalité, ce risque est très faible. Cone et coll. (37) ont montré qu'en utilisant un seuil de positivité à $20 \mathrm{ng} / \mathrm{ml}$, des sujets placés dans une pièce étroite $(2,1 \mathrm{~m} \times 2,5 \mathrm{~m} \mathrm{x} \mathrm{2,4} \mathrm{m})$ et non ventilée, étaient positifs après avoir été exposés à la fumée de 16 cigarettes contenant $2,8 \%$ de THC une heure par jour pendant 6 jours consécutifs. D'autres auteurs (38) ont confirmé ces données en utilisant les mêmes conditions d'exposition, mais dans une pièce de $30 \mathrm{~m}^{3}$ : les concentrations maximales urinaires observées chez les sujets exposés à la fumée de cannabis étaient de $4,4 \mathrm{ng} / \mathrm{ml}$ donc largement inférieures au seuil de positivité de $50 \mathrm{ng} / \mathrm{ml}$.

Pour les laboratoires ayant une faible fréquence de demandes ou pour une utilisation sur le terrain (accidents de la voie publique), certaines sociétés ont commercialisé des tests rapides faisant appel à l'immunochromatographie, présentés sous forme de bandelettes réactives. La plupart de ces tests ont été conçus pour l'urine, les anticorps étant dirigés contre le THC$\mathrm{COOH}$, et certains d'entre eux possèdent des performances très satisfaisantes. Ils ne sont en aucun cas utilisables pour des milieux biologiques tels que la sueur ou la salive, dans lesquels seul le THC peut être présent (39). Récemment des tests rapides ont été développés pour une utilisation dans la salive, les anticorps étant dirigés contre le THC. Cependant, les seuils de positivité sont largement supérieurs à ceux qui sont observés dans la salive. En effet, la sensibilité nécessaire pour mettre en évidence un usage de cannabis est de $4 \mathrm{ng} / \mathrm{ml}$ de salive. Or, les tests précités permettent seulement de détecter des concentrations comprises entre 40 et $100 \mathrm{ng} / \mathrm{ml}$, ce qui est encore très éloigné des performances requises (40).

\section{Les méthodes de confirmation et' de dosage font obli-} gatoirement appel à des techniques analytiques séparatives et spécifiques. De très nombreuses méthodologies ont été proposées, mais la chromatographie gazeuse couplée à la spectrométrie de masse (GS-MS) est actuellement la plus utilisée. La méthode proposée par Kintz et coll. en 1996 (41) figure parmi les méthodes les plus performantes, pour des concentrations en cannabinoïdes égales ou supérieures à $1 \mathrm{ng} / \mathrm{ml}$. Des études en cours devraient permettre de proposer des méthodes plus adaptées pour des concentrations inférieures.
L'interprétation des résultats, relativement aisée pour d'autres stupéfiants, est complexe avec les cannabinoïdes, en raison de l'existence de 2 principes actifs et du comportement toxicocinétique très particulier du THC. Le principal problème est qu'il n'existe pas de corrélation entre les concentrations sanguines en THC et les effets sur le consommateur (16), du fait de sa fixation tissulaire rapide et intense. Des travaux préliminaires réalisés récemment chez le porc et chez l'homme (14) ont montré que le THC pouvait encore être présent dans le cervelet, le cortex occipital et le cortex frontal à des concentrations significatives alors qu'il n'était plus détectable dans le compartiment sanguin.

Une étude rétrospective a permis à Daldrup (42) de proposer une relation entre les concentrations en cannabinoïdes et le degré d'altération neuro-comportementale du sujet. Le CIF (Cannabis Influence Factor) est déterminé ainsi :

$\mathrm{CIF}=[\mathrm{THC} / 314,5+11-\mathrm{OH}-\mathrm{THC} / 330,5] /[\mathrm{THC}-$ $\mathrm{COOH} \times 0,01 / 344,5]$

Lorsque la valeur du CIF est égale ou supérieure à 10 , il considère que les fonctions cognitives et motrices sont perturbées. Cependant, cette formule n'a à ce jour jamais été validée par d'autres auteurs.

Huestis et coll (17) ont déterminé un modèle mathématique prenant en compte les concentrations sanguines de THC et de THC-COOH, permettant d'estimer le temps écoulé entre le moment de la dernière consommation et celui de la prise de sang avec un intervalle de confiance de $92 \%$. Ce modèle était le suivant :

$\log \mathrm{T}($ en $\mathrm{h})=[0,576 \times \log (\mathrm{THC}-\mathrm{COOH} / \mathrm{THC})]-$ 0,176

Cette formule est dans la pratique difficilement utilisable, car ayant été établie avec des administrations uniques, elle n'est pas adaptée pour des consommations régulières ou répétées.

A la lumière de tous ces éléments et dans l'attente de travaux complémentaires, la seule alternative restant à ce jour est donc de considérer que la présence dans le sang de THC et/ou 11-OH-THC au dessus de la limite de détection $(0,2$ à $0,4 \mathrm{ng} / \mathrm{ml}$ selon la méthode utilisée) permet d'estimer que le sujet était sous influence de cannabis au moment du prélèvement. La présence de THC dans le sang peut être exceptionnellement observée de nombreuses heures voire plusieurs jours après consommation. Bien qu'aucune étude n'ait été effectuée à ce jour sur ce thème, il est fort probable que cela puisse s'expliquer par un phénomène de relargage tissulaire. Une telle hypothèse n'est pas sans fondement scientifique, des auteurs ayant détecté des cannabinoïdes dans des biopsies de tissus adipeux 4 semaines après la dernière consommation de cannabis (43). 


\section{Conclusion}

Considérée par certains comme une «drogue douce», Cannabis sativa var. indica est une plante dont le principal principe actif, le $\mathrm{THC}$, possède en réalité une toxicité aiguë et chronique non négligeable, se traduisant par des effets somatiques et psychiques importants. Les nouvelles variétés de plantes à forte teneur en THC permettent de classer le cannabis parmi les plantes à pouvoir hallucinogène. Les conséquences de son usage pour autrui et notamment dans le domaine de l'insécurité routière (44) ont conduit de nombreux pays dont la France (45) à mettre en place une législation visant à sanctionner les conducteurs ayant fait usage de cannabis. Compte tenu d'un nombre de consommateurs de plus en plus important et en particulier dans la population active, le dépistage obligatoire de sa consommation chez les personnes occupant des postes à risque et/ou de sécurité dans les entreprises apparaît désormais être une nécessité.

Au cours de ces dernières années, le cannabis a été l'objet de très nombreuses études et nos connaissances sur les mécanismes d'action et la physiopathologie du cannabis ont énormément progressé. Cependant, de nombreuses questions se posent encore dans divers domaines : distribution et redistribution tissulaire des cannabinoïdes, toxicité induite par les produits associés dans la fabrication de la «résine», etc. Pour y répondre, les études chez l'homme sont peu réalisables pour des raisons évidentes d'éthique et les travaux réalisés sur les rongeurs (27) ont clairement montré que ces animaux constituaient de très mauvais modèles pour ce produit. En conséquence, des études effectuées sur des mammifères comme le porc pourraient être envisagées et devraient permettre de répondre à bon nombre de ces questions.

\section{Références}

1. Richard D., Senon J.L. Le cannabis. Paris : Presses Universitaires de France, 1996.

2. Chollet-Przednowed E. Cannabis : le dossier. Paris : Gallimard, 2003.

3. Moreau J. Du haschich et de l'aliénation mentale. Paris : De Fortin et Masson ; 1845.

4. ESCAPAD 2001. Santé, mode de vie et usages de drogues à 18 ans. OFDT, Paris, juin 2002.

5. Bruneton J. Cannabaceae. In : Bruneton J, eds. Plantes toxiques. Paris : Lavoisier, $1996: 210-4$.

6. Mathieu J.P. Chanvre. Techniques agricoles $1980 ; 2130$ (5) : 1-10.

7. Fournier G., Richez-Dumanois C., Duvezin J., Mathieu J.P., Paris M. Identification of a new chemotype in Cannabis sativa: cannabigerol-dominant plants, biogenetic and agronomic prospects. Planta Med. 1987 ; 53 : 277-80.
8. Règlement (CE) $\mathrm{n}^{\circ} 2860 / 2000$ de la Commission du 27 décembre 2000. Journal Officiel des Communautés Européennes du 28 décembre 2000.

9. Mura P., Perrin M., Chabrillat M., Chaudron H., Dumestre-Toulet V., Barc S., Pépin G. L'augmentation des teneurs en delta-9-tétrahydrocannabinol dans les produits à base de cannabis en France : mythe ou réalité ? Ann. Toxicol. Anal. $2001 ; 13: 75-9$.

10. Mura P., Piriou A. Cannabis. In : Kintz P ed. Toxicologie et pharmacologie médicolégales. Paris : Elsevier, 1998, 543-54.

11.Pelissier A.L., Leonetti G., Villani P., Cianfarani F., Botta A. Cannabis: toxicokinetic focus and methodology of urinary screening. Thérapie $1997 ; 52: 213-8$.

12. Huestis M.A., Sampson A.H., Holicky B.J., Henningfield J.E., Cone E.J.. Characterization of the absorption phase of marijuana smoking. Clin. Pharmacol. Ther. 1992 ; $52: 31-41$.

13. Huestis M.A., Henningfield J.E., Cone E.J.. Blood cannabinoids. I. Absorption of THC and formation of 11$\mathrm{OH}-\mathrm{THC}$ and $\mathrm{THCCOOH}$ during and after smoking marijuana. J. Anal. Toxicol. 1992 ; 16 : 276-82.

14. Mura P., Brunet B., Dumestre V., Pépin G., Kintz P., Papet Y., Hauet T. Comparaison entre les concentrations sanguines et cérébrales de THC chez le porc et chez l'homme : incidences dans le domaine de la conduite automobile après usage de cannabis. Troisièmes ateliers européens de pharmacodépendance. Biarritz, France, 2728 octobre 2003. In : http://www.centres-pharmacodependance.net/Congres/biarritz

15. Baselt R.C., Cravey R.H. Tetrahydrocannabinol. In : Baselt RC and Cravey RH eds. Disposition of toxic drugs and chemicals in man. Foster City : Chemical Toxicology Institute, $1995: 713-7$.

16. Mura P., Brunet B., Monzon C., Papet Y., Mauco G. Interprétation des concentrations sanguines en cannabinoïdes : données nouvelles. Ann. Toxicol. Annal. 2003 ; $15: 35-9$.

17. Huestis M.A., Henningfield J.E., Cone E.J. Blood cannabinoids. II. Models for the prediction of time of marijuana exposure from plasma concentrations of Delta9- tetrahydrocannabinol (THC) and 11-nor-9-carboxy-Delta9tetrahydrocannabinol (THCCOOH). J. Anal. Toxicol. 1992 ; $16: 283-90$.

18.Stillman R., Galanter M., Lemberger L. Tetrahydrocannabinol (THC): metabolism and subjective effects. Life Sci. $1976 ; 19: 569-76$.

19. Barnett G., Licko V., Thompson T. Behavioral pharmacokinetics of marijuana. Psychopharmacol. 1985 ; 85 : 51-6.

20. Cochetto D.M., Owens S.M., Perez-Reyes M., DiGuiseppi S., Miller L.L. Relationship between plasma delta-9-tetrahydrocannabinol concentration and pharmacologic effects in man. Psychopharmacol. $1981 ; 75$ : 158-64.

21. Harder S., Rietbrock S. Concentration-effect relationship of (-9-tetrahydrocannabinol and prediction of psychotropic effects after smoking marijuana. Int. J. Clin. Pharmacol. and Therapeutics 1997 ; 35 : 155-9.

22. Schou J., Prockop L.D., Dahlstrom G., Rohde C. Penetration of delta-9-tetrahydrocannabinol and 11-OHdelta-9-tetrahydrocannabinol through the blood-brain barrier. Acta Pharmacol. Toxicol. 1977 ; 41 33-8. 
23. Kelly P., Jones R.T. Metabolism of tetrahydrocannabinol in frequent and infrequent marijuana users. J. Anal. Toxicol. $1992 ; 16: 228-35$.

24. Garrett E.R. Pharmacokinetics and disposition of delta-9tetrahydrocannabinol and its metabolites. In : Marijuana : Biological effects (Nahas G.G., Paton W.D.M., eds). New York : Pergamon Press, 1979, 10521.

25. Huestis M.A., Cone E.J. Urinary excretion half-life of 11-nor-9-carboxy-Delta9- tetrahydrocannabinol in humans. Ther. Drug Monit. 1998 ; 20 : 570-6.

26. Smith-Kielland A., Skuterud B., Morland J. Urinary excretion of 11-nor-9-carboxy-delta9-tetrahydrocannabinol and cannabinoids in frequent and infrequent drug users. J. Anal. Toxicol. $1999 ; 23$ : 323-32.

27. Expertise collective INSERM. Cannabis. Quels effets sur le comportement et la santé ? In : INSERM, ed. Paris : Jouve, 2001

28. Karila L., Reynaud M. Cognitive disorders and chronic use of cannabis. Ann. Med. Interne $2003 ; 154$ Spec No $1:$ S58-64.

29. Council on Scientific Affairs. Marijuana : its health hazards and therapeutic potentials. JAMA $1981 ; 246$ : 1823-7.

30. Bech P., Rafaelsen L., Rafaelsen O.J. Cannabis and alcohol: effects on estimation of time and distance. Psychopharmacologia $1973 ; 32: 373-81$.

31. Kurzthaler I., Hummer M., Miller C., SpernerUnterweger B., Gunther V., Wechdorn H., Battista H.J., Fleichhacker W.W. Effect of cannabis use on cognitive functions and driving ability. J. Clin. Psychiatry 1999 ; $60: 395-9$.

32. Park B., McPartland J.M., Glass M. Cannabis, cannabinoids and reproduction. Prostaglandins Leukot. Essent. Fatty Acids $2004 ; 70$ (2) : 189-97.

33. Kaminsky N.E. Mechanisms of immune modulation by cannabinoids. In : Dean JH, Luster MI, Munson AE, Kimber I, eds. Immunotoxicology and Immunopharmacology. New York : Raven Press, 1994 : 349-62.

34. Richard D., Senon J.L. Le cannabis. Paris : Presses Universitaires de France, 1996.
35. Smit F., Bolier L., Cuijpers P. Cannabis use as a probable causative factor in the later development of schizophrenia. Ned Tijdschr Geneeskd 2003 ; 147 : 2178-83.

36. Dervaux A., Laqueille X., Bourdel M.C., Leborgne M.H., Olie J.P., Loo H., Krebs M.O. Cannabis and schizophrenia : demographic and clinical correlates. Encéphale $2003 ; 29: 11-7$.

37. Cone E.J., Johnson R.E., Darwin W.D., Yousefnej A.D., Mell L.D., Paul B.D., Mitchell J. Passive inhalation of marijuana smoke : urinalysis and room air levels of delta9-tetrahydrocannabinol. J. Anal. Toxicol. $1987 ; 11$ : 8996.

38. Giardino N.J. An indoor air quality-pharmacokinetic simulation of passive inhalation of marijuana smoke and the resultant buildup of 11-nor-delta-9-tetrahydrocannabinol-9-carboxylic acid in urine. J. Forensic Sci. 1997 ; $42: 323-5$.

39. Mura P., Kintz P., Papet Y., Ruesch G., Piriou A. Evaluation de six tests rapides pour le dépistage du cannabis dans la sueur, la salive et les urines. Acta Clin. Belg. 1999 ; 1 : 35-8.

40. Verstraete A., Samyn N. Le dépistage biologique d'une conduite sous influence. Ann. Tox. Annal. 2003 ; 15 : 83-91.

41. Kintz P., Cirimele V., Pepin G., Marquet P., Deveaux M., Mura P. Identification et dosage des cannabinoïdes dans le sang total. Toxicorama $1996 ; 8: 29-33$.

42. Daldrup T. Cannabis Influence Factor. Congrès de I'International Association of Forensic Sciences, Tokyo, 1996.

43. Johansson E., Noren K., Sjovall J., Halldin M.M. Determination of delta-1-tetrahydrocannabinol in human fat biopsies from marihuana users by gas chromatography-mass spectrometry. Biomed. Chromatogr. $1989 ; 3$ : 35-8.

44. Mura P., Kintz P., Ludes B., Gaulier J.M., Marquet P., Martin-Dupont S., Vincent F., Kaddour A., Goullé J.P., Nouveau J., Moulsma M., Tilhet-Coartet S., Pourrat O. Comparison of the prevalence of alcohol, cannabis and other drugs between 900 injured drivers and 900 control subjects : results of a French collaborative study. Forensic Sci. Int. $2003 ; 133: 79-85$.

45. Journal Officiel de la République Française, 4 février 2003, p. 2103. 